\title{
High-Grade B-Cell Lymphoma (HGBL) with MYC and BCL2 and/or BCL6 Rearrangements Is Predominantly BCL6-Rearranged and BCL6-Expressing in Taiwan
}

\author{
Cheng-Chih Tsai ${ }^{1,+}+\mathbb{C}$, Yung-Cheng Su ${ }^{1,2, t, \ddagger}$, Oluwaseun Adebayo Bamodu ${ }^{1,3,+} \mathfrak{\infty}$, Bo-Jung Chen ${ }^{4}$, \\ Wen-Chiuan Tsai ${ }^{5}$, Wei-Hong Cheng ${ }^{1}$, Chii-Hong Lee ${ }^{4,6}$, Shu-Min Hsieh ${ }^{7}$, Mei-Ling Liu ${ }^{4}$, Chia-Lang Fang ${ }^{8}$, \\ Huan-Tze Lin ${ }^{9}$, Chi-Long Chen ${ }^{8,10}{ }^{\mathbb{D}}$, Chi-Tai Yeh 1,3 ${ }^{\mathbb{D}}$, Wei-Hwa Lee ${ }^{4}$, Ching-Liang Ho ${ }^{11}$, Shiue-Wei Lai ${ }^{11,12}$, \\ Huey-En Tzeng ${ }^{9,13}$, Yao-Yu Hsieh ${ }^{1}$, Chia-Lun Chang ${ }^{14}$, Yu-Mei Zheng ${ }^{14}$, Hui-Wen Liu ${ }^{1}{ }^{1}$, Yun Yen ${ }^{13}$, \\ Jacqueline Whang-Peng ${ }^{14,15}$ and Tsu-Yi Chao ${ }^{1,11,12,15, *}$
}

check for updates

Citation: Tsai, C.-C.; Su, Y.-C.;

Bamodu, O.A.; Chen, B.-J.; Tsai, W.-C.; Cheng, W.-H.; Lee, C.-H.; Hsieh, S.-M.; Liu, M.-L.; Fang, C.-L.; et al. High-Grade B-Cell Lymphoma (HGBL) with MYC and BCL2 and/or BCL6 Rearrangements Is Predominantly BCL6-Rearranged and BCL6-Expressing in Taiwan. Cancers 2021, 13, 1620. https://doi.org/10.3390/ cancers 13071620

Academic Editors: Alexey Ushmorov and Chalid Assaf

Received: 15 January 2021

Accepted: 26 March 2021

Published: 31 March 2021

Publisher's Note: MDPI stays neutral with regard to jurisdictional claims in published maps and institutional affiliations.

Copyright: (c) 2021 by the authors. Licensee MDPI, Basel, Switzerland. This article is an open access article distributed under the terms and conditions of the Creative Commons Attribution (CC BY) license (https:// creativecommons.org/licenses/by/ $4.0 /)$.
1 Division of Hematology and Oncology, Department of Internal Medicine, Taipei Medical University-Shuang Ho Hospital, New Taipei City 235, Taiwan; minehoks@gmail.com (C.-C.T.); xxxx@s.tmu.edu.tw (Y.-C.S.); 16625@s.tmu.edu.tw (O.A.B.); 13520@s.tmu.edu.tw (W.-H.C.); ctyeh@s.tmu.edu.tw (C.-T.Y.); 10573@s.tmu.edu.tw (Y.-Y.H.); 19290@s.tmu.edu.tw (H.-W.L.)

2 Ph.D. Program for Cancer Molecular Biology and Drug Discovery, College of Medical Science and Technology, Taipei Medical University and Academia Sinica, Taipei City 115, Taiwan

3 Department of Medical Research and Education, Taipei Medical University-Shuang Ho Hospital, New Taipei City 235, Taiwan

4 Department of Pathology, Taipei Medical University-Shuang Ho Hospital, New Taipei City 235, Taiwan; 15006@s.tmu.edu.tw (B.-J.C.); b8301130@tmu.edu.tw (C.-H.L.); s20021@skmh.tmu.edu.tw (M.-L.L.); whlpath97616@shh.org.tw (W.-H.L.)

5 Department of Pathology, Tri-Service General Hospital, National Defense Medical Center, Taipei City 114, Taiwan; doc31779@mail.ndmctsgh.edu.tw

6 Department of Anatomic Pathology, Taipei Institute of Pathology, Taipei City 103, Taiwan

7 Department of Clinical Pathology, Taipei Medical University-Shuang Ho Hospital, New Taipei City 235, Taiwan; 13464@s.tmu.edu.tw

8 Department of Pathology, Taipei Medical University Hospital, Taipei City 110, Taiwan; ccllfang@tmu.edu.tw (C.-L.F.); chencl@tmu.edu.tw (C.-L.C.)

9 Division of Hematology and Oncology, Department of Medicine, Taipei Medical University Hospital, Taipei City 110, Taiwan; mount591@hotmail.com (H.-T.L.); tzhuen@tmu.edu.tw (H.-E.T.)

10 Department of Pathology, School of Medicine, Taipei Medical University, Taipei City 110, Taiwan

11 Division of Hematology-Oncology, Department of Internal Medicine, Tri-Service General Hospital, National Defense Medical Center, Taipei City 114, Taiwan; 02241@ndmctsgh.edu.tw (C.-L.H.); xsurfer@office365.ndmctsgh.edu.tw (S.-W.L.)

12 Graduate Institute of Clinical Medicine, College of Medicine, Taipei Medical University, Taipei City 110, Taiwan

13 Program for Cancer Molecular Biology and Drug Discovery, College of Medical Science and Technology, Taipei Medical University, Taipei City 110, Taiwan; yyen@tmu.edu.tw

14 Department of Medicine, Division of Hematology and Oncology, Taipei Medical University-Wan-Fang Hospital, Taipei City 116, Taiwan; richardch9@tmu.edu.tw (C.-L.C.); lilindr3@gmail.com (Y.-M.Z.); jqwpeng@nhri.org.tw (J.W.-P.)

15 Taipei Cancer Center, Taipei Medical University, Taipei City 110, Taiwan

* Correspondence: 10575@s.tmu.edu.tw; Tel.: +886-2-2249-0088 (ext. 8402); Fax: +886-2-6639-7181

+ These authors contributed equally to this work.

$\ddagger$ Deceased.

Simple Summary: This study highlights the epidemiological, cytogenetic and clinical difference between patients with multiple hit diffuse large B-cell lymphoma in Taiwan and those from western countries. Unlike in the West, the majority of patients with multiple hit lymphoma in Taiwan harbor a BCL6 rearrangement. Almost three in every five BCL6-rearranged double hit lymphoma cases in Taiwan are non-GCB phenotype, indicating, at least in part, that the preferential screening for double hit with $B C L 6$ rearrangement may be a clinically-informative modality for patients with non-GCB phenotype DLBCL in Taiwan. This also suggests the need for a different treatment approach than is obtained in the West where BCL6 double hit lymphomas are seemingly GCB. Consistent with our 
present findings, mandatory screening for BCL6-rearrangement in suspected DLBCL cases in Taiwan may aid early diagnosis, therapy decision, and clinical outcome forecast.

Abstract: This study investigated the epidemiological and clinical peculiarities of BCL2 and BCL6 rearrangement in patients with high grade B-cell lymphoma (HGBL) from Taiwan, compared with data from Western countries. Two hundred and eighty-two DLBCL cases from Taipei Medical University-affiliated hospitals $(n=179)$ and Tri-Service General Hospital $(n=103)$ were enrolled for this study. From the 282, 47 (16.7\%) had MYC translocation; 24 of these harbored concurrent $B C L 2$ and/or BCL6 translocation (double-hit, DH or triple-hit, TH). Twelve DH-HGBL cases had simultaneous $M Y C$ and BCL6 translocations, 8 harbored MYC and BCL2 rearrangement, while the remaining 4 patients exhibited TH. Together, $66.7 \%$ of DH/TH-HGBL patients were BCL6 rearrangement positive. Among these BCL6-rearranged DH/TH-HGBL patients, only 6 (37.5\%) overexpressed MYC and BCL6 proteins simultaneously, indicating that MYC-BCL6 co-overexpression may not be plausible surrogate biomarker for screening BCL6-rearranged DH-HGBL. By the end of year 5, all patients with TH-HGBL, BCL2 DH-HGBL and all but one BCL6 DH-HGBL cases had expired or were lost to follow-up. Progression-free survival (PFS) was longer for the non-DH/THHGBL group compared with the DH/TH-HGBL group. While the patients with BCL2 DH-HGBL were lost to follow-up by day 800, their remaining TH-HGBL and BCL6 DH-HGBL peers exhibited very poor PFS, regardless of age strata. More so, patients with BCL6 rearrangement were 5.5-fold more likely associated with extranodal involvement compared with their BCL2-rearranged peers. Moreover, $\sim 60.0 \%$ of the BCL6-rearranged DH-HGBL cases were non-GCB, suggesting that including screening for $B C L 6$ rearrangement in patients with the non-GCB phenotype may aid medical decisionmaking and therapeutic strategy. Contrary to contemporary data from western countries, 2 in every 3 patients with DH/TH-HGBL in Taiwan harbor BCL6 rearrangement. Consistent with present findings, we recommend mandatory screening for $B C L 6$ rearrangement in patients with aggressive HGBL in Taiwan.

Keywords: MYC; BCL2; BCL6; HGBL; DLBCL; double-hit lymphoma; non-GCB; gene rearrangement; non-Hodgkin's lymphoma

\section{Introduction}

Diffuse large B-cell lymphoma (DLBCL) is the most common subtype of non-Hodgkin's lymphoma and it accounts for about half of all lymphomas in Taiwan [1]. DLBCL is composed of a heterogeneous group of morphologically similar lymphomas with a broad prognostic spectrum. Clinical prognostication is mostly aided by gene expression profiling (GEP). Based on GEP, DLBCL can be divided into 3 biologically distinct subtypes based on cell-of-origin (COO), namely germinal center B-cell (GCB), activated B-cell (ABC) and type III [2].

The last two decades has been characterized by piqued interest in identifying and demystifying the unique subtypes of DLBCL COO, alongside the molecular features that may facilitate their use, independent of the International Prognostic Index (IPI), for patient stratification, high-risk disease group identification, and prediction of treatment failure and/or prognosis [3]. Limitations in the clinical application/adoption of the GEP-based COO identification, due to its high cost and mandatory requirement of fresh frozen tissue, has led to the use of immunohistochemisty (IHC)-based methods like the Tally and Hans algorithms for $\mathrm{COO}$ identification in clinical practice [3]. Howbeit, compared with GEP (the gold standard for identification of COO), the sensitivity of $\mathrm{IHC}$ is about $70 \%$ and $90 \%$ for the GCB and so-called "non-GCB" groups, respectively [3,4]. More recently, there has been development of new platforms, like the RNA-based Lymph2Cx assay, which are yet to be adapted for clinical use but exhibit relatively higher concordance with GEP than the IHC, are reproducible across laboratories, and are adaptable for digital GEP of fixed 
paraffin-embedded tissue [4-6]. Furthermore, apart from the COO classification of DLBCL, another RNA-based method called the comprehensive consensus clustering (CCC), which identifies prevalent B-cell receptor signaling-associated metabolic pathways and the crucial peculiarities in tumor immune/inflammatory infiltrate, has been touted for identification of distinct subtypes of DLBCL [7]. However, despite the ability of the CCC to identify important intra-DLBCL heterogeneity, its clinical application remains limited.

Moreover, in an effort to provide "a potential nosology for precision-medicine strategies in DLBCL", Schmitz et al. reported the discovery of four principal "genetic subtypes of DLBCL with distinct genotypic, epigenetic, and clinical characteristics", namely, MCD (characterized by co-occurrence of $M Y D 88^{\mathrm{L} 265 \mathrm{P}}$ and $C D 79 B$ mutations), and $\mathrm{N} 1$ (based on NOTCH1 mutations) which are ABC-dominated, the EZB (rich in EZH2 mutations and $B C L 2$ translocations) which is mostly GCB, and the BN2 (harboring BCL6 fusions and $\mathrm{NOTCH} 2$ mutations) consisting of $\mathrm{ABC}, \mathrm{GCB}$ and unclassified cases [8]. In spite of the advances in biomolecular techniques that have significantly helped to expand our understanding of the pathobiology of DLBCL, facilitating the identification of disease subsets with similar targetable bio-traits, the GEP remains the gold standard for identification of COO. While the GCB DLBCL is characterized by good prognosis with a 5-year overall survival (OS) of $\sim 59 \%$, the ABC DLBCL is more aggressive and associated with very poor prognosis when treated with standard chemotherapy, with a 5-year OS of $\sim 31 \%[9,10]$.

There is also accrued evidence that DLBCL with concurrent rearrangement of MYC and $B C L 2$ or $B C L 6$, (also called double-hit lymphoma (DHL)) are characterized by dismal prognosis, as evidenced by median OS $<1.5$ years, regardless of $\mathrm{COO}$ [11]. Until date, conventional knowledge indicates that majority (90-95\%) of DHL are GCB phenotype, and that while concurrent $B C L 2 / M Y C$ rearrangement accounts for $\geq 75 \%$ of DHL, the less likely $B C L 6$-rearranged DHL accounts for $<25 \%[11,12]$.

It has also been reported that most BCL2-rearranged DHL have concurrent overexpression of MYC and BCL2 detected by immunohistochemical (IHC) staining [13]. However, while the clinicopathological characteristics and probable therapeutic options of BCL2rearranged DHL are well studied and documented; only few studies have focused on BCL6-rearranged DHL and its clinical features. In one of those few studies, Li et al. in their clinicopathological analysis of DHL, found 13 BCL6-rearranged and 83 BCL2-rearranged DHL cases from a DLBCL cohort of $\sim 1000$ patients, however they reported no dissimilarity in their clinicopathological characteristics, except that the BCL6-rearranged DHL was less likely to be phenotypically GCB [14]. The dearth of documented consensual clinicopathological characterization of Taiwanese patients with DHL/THL, otherwise designated highgrade B-cell lymphoma (HGBL) with MYC and BCL2 and/or BCL6 rearrangements [14,15], informs the present study. Thus, the present study (i) investigated the epidemiological peculiarity of patients with DHL in Taiwan, (ii) comparatively analyzed the cytogenetic and clinical traits of DHL cases in Taiwan and reported cohort traits from western countries, and (iii) delineates the clinicopathological characteristics with associated prognostic peculiarity of patients with DHL in Taiwan. The present study incorporates patients with DLBCL from Shuang Ho Hospital, Taipei Medical University Hospital and Wan-Fan Hospital as cohort 1, and Tri-Service General Hospital as cohort 2. Interestingly, contrary to contemporary data mostly originating from western countries, in Taiwan, the majority $(68.2 \%)$ of patients with DH/TH-DLBCL harbor BCL6 rearrangement, and MYC/BCL6 co-expression is not a surrogate marker of MYC/BCL6-rearranged DH/TH-DLBCL.

\section{Methods}

\subsection{Sample Selection}

A total of 282 patients with DLBCL diagnosed between January, 2009 and December, 2019 and with plausible cytogenetic and corresponding clinicopathological data were selected from Shuang Ho Hospital, Taipei Medical University Hospital, Wan-Fang Hospital, and Tri-Service General Hospital. In our study consortium, almost all new cases of DLBCL were tested for MYC rearrangements by fluorescence in-situ hybridization (FISH) analysis, 
and MYC-rearranged cases were further probed for BCL2 or BCL6 rearrangements. Initial classification of cases was based on the 2008 World Health Organization (WHO) classification of hematopoietic and lymphoid tissues [16], and re-classified consistent with the 2016 WHO classification guideline [17] by 2 experienced hematopathologists. No tumor specimen was positive for Epstein-Barr virus (EBV) infection. DLBCL with MYC and BCL2 and/or BCL6 rearrangements per the classic definition were defined as DHL/THL, and those with co-expression of MYC and BCL2 and/or BCL6 protein were defined as double or triple expressor lymphoma (DEL/TEL). Patients' clinicopathological information was retrieved from digital medical records of the hematopathological departments of participating medical centers; The attending physicians determined therapy regimen for the patients, and this included R-CHOP (rituximab, cyclophosphamide, doxorubicin, vincristine, and prednisone) with or without etoposide, or R-EPOCH (rituximab, etoposide, prednisone, vincristine, cyclophosphamide, and doxorubicin).

\subsection{Fluorescence In Situ Hybridization (FISH)}

MYC, BCL2 or BCL6 translocation was detected by the FISH, using the break-apart probes of target genes, strictly following manufacturer's instruction. We tested all samples with the MYC probe first, followed by screening for BCL2 and BCL6 translocations in samples harboring MYC translocation. The probes, namely Vysis LSI MYC (8q24.21) (Cat\# 05J91-001), Vysis LSI BCL2 (18q21.33) (Cat\# 07J75-001), and Vysis LSI BCL6 (3q27.3) (Cat\# 01N23-020) (ASR) dual color break apart rearrangement probes, were purchased from Abbott Laboratories (Chicago, IL, USA). As previously described [18], MYC, BCL2 and BCL6 were defined as rearranged when they exhibited the appearance of disjointed individual green signal and red signal; For every sample, the probe signals for monolayers of $\geq 200$ DLBCL cell nuclei were counted under fluorescence microscope at $\times 100$ magnification, and probe signals exceeding $20 \%$ threshold in the number of nuclei was considered genetic alterations.

\subsection{Immunohistochemical (IHC) Staining}

IHC staining was performed to evaluate the expression level of MYC, BCL2 or BCL6 proteins in our DLBCL samples, using $3 \mu \mathrm{m}$ formalin-fixed paraffin-embedded (FFPE) tissue sections which were subjected to $3 \mathrm{~min}$. Ethylenediaminetetraacetic acid (EDTA) buffer-based heat-induced antigen retrieval. The samples were probed with monoclonal primary antibodies against CD10 (clone 56C6; Cat\# MA5-14050, 1:5, ThermoFisher Scientific, Carlsbad, CA, USA.), IRF4/MUM1 (clone EP190; Cat\# BSB-6958, 1:80, BioSB, Santa Barbara, CA, USA), BCL2 (RTU clone SP66; Cat\# 790-4604, Roche Tissue Diagnostics, Oro Valley, AZ, USA), MYC (clone EP121; Cat\# BSB-6581, 1:30, BioSB), BCL6 (RTU clone GI191E/A8; Cat\# 760-4241, Roche Tissue Diagnostics), and Ki67 (clone SP6; Cat\# MA5-14520, 1:200, ThermoFisher Scientific). All staining was performed using the Ventana benchmark ULTRA IHC staining module (Ventana, Tucson, AZ, USA). As previously described [19], for COO determination based on Hans' algorithm, positivity cut-off for CD10, IRF4/MUM1, and BCL6 expression was $\geq 30 \%$ stained cells, while the expression positivity cut-off for BCL2 or MYC was $\geq 50 \%$ or $\geq 40 \%$ of stained cells, respectively.

\subsection{Statistical Analysis}

Correlative analysis and determination of nonrandom association between gene rearrangement or expression and patients' clinicopathological features were performed by chi-squared $\left(\chi^{2}\right)$ or Fisher exact tests. If the test statistic exceeds the critical value of $\chi^{2}$, the null hypothesis ( $H_{0}=$ there is no difference between the distributions) can be rejected, and the alternative hypothesis $\left(H_{1}=\right.$ there is a difference between the distributions) can be accepted with the selected level of confidence; in order words we can say the variables are dependent/related. Thus, Prob $>\chi^{2}$ implies the probability that the test statistic exceeds the critical value of $\chi^{2}$ and allows us make the judgement that the null hypothesis can be rejected and the alternative hypothesis accepted, establishing the association between 
the variables, i.e., the translocation status (BCL2 DHL, BCL6 DHL, THL) is related to or associated with survival status. It is important then, that the Prob $>\chi^{2}$ and $\chi^{2}$ be interpreted in the context of each other, not as standalone values. The overall survival (OS), defined as the period from diagnosis to day of death or last follow-up and progression-free survival (PFS), defined as the period from primary treatment to day of disease worsening and/or recurrence, were estimated using Kaplan-Meier (KM) plots, and the inter-group comparison of survival differences was evaluated using the log-rank test. Prognostic relevance was determined using univariate and multivariate Cox regression models. Where applicable, compensation for missing data was achieved using the stochastic regression imputation method. All statistical analyses were performed with IBM SPSS Statistics for Windows, Version 20.0 (IBM Corp., Armonk, NY, USA). A p-value < 0.05 was considered statistically significant.

\section{Results}

\subsection{Cohort Clinical Characteristics, Gene Rearrangement, and Protein Expression}

Our cohort of 282 patients with DLBCL consists of 162 males and 120 females with a median age of $66.5 \pm 15.2$ years. 47 (16.7\%) patients harbored MYC rearrangement, while 235 (83.3\%) exhibited no MYC rearrangement. Of the MYC-rearranged cases, 33\%, $50 \%$, and $\sim 17 \%$ concurrently bore BCL2, BCL6, or BCL2+BCL6 rearrangement, respectively (Figure 1). Based on COO, 116 (41.1\%) of the total cohort were GCB-type, while 166 (58.9\%) were non-GCB-type DLBCL (Table 1). Among the 282 patients, 47 (16.7\%) had MYC translocation, and of these $47 M Y C$-rearranged cases, concurrent BCL2 and/or BCL6 translocation were detected in 24 patients; Half of them (12 patients) had simultaneous MYC and BCL6 translocations, eight harbored $M Y C$ and $B C L 2$ rearrangements, while the remaining four patients exhibited a triple hits (TH) genotype with concurrent MYC, BCL2 and BCL6 translocations (Figure 1, Table 2). Together, 16 (66.7\%) of DH/TH-HGBL patients were positive for BCL6 rearrangement (Figure 1). Among these DH/TH-HGBL patients with BCL6 rearrangement, only six (37.5\%) overexpressed MYC and BCL6 proteins simultaneously as shown by IHC staining, indicating the co-overexpression of MYC and BCL6 proteins may not be a plausible surrogate biomarker for screening BCL6-rearranged DH HGBL (Table 2). Moreover as shown in Table 2, nine of sixteen (56.3\%) BCL6-rearranged DH/THHGBL cases, and $58.3 \%$ of the BCL6-rearranged DH-HGBL cases were non-germinal center B-cell (non-GCB), while six of 12 (50.0\%) patients with BCL6-rearranged DHL had Ann Arbor stages III/IV disease, suggesting that added screening for DH/TH-DLBCL with $B C L 6$ rearrangement in patients with the non-GCB phenotype of DLBCL may aid medical decision-making and therapeutic strategy.

3.2. The Relationship between MYC, BCL2, and BCL6 Rearrangement, Expression and Clinicopathological Features

\subsubsection{MYC, BCL2, BCL6, and COO}

Compared to patients with BCL2 DHL, those with BCL6 DHL were more associated with MUM1 expression (25.0\% vs. 66.7\%). Conversely, the BCL2 DHL group was more likely to express CD10/MME protein in comparison with the BCL6 DHL group (87.5\% vs. $50.0 \%$ ). Consistently, almost three out of every five BCL6 DHL cases (58.3\%) exhibited the non-GCB phenotype, while the BCL2 DHL group (87.5\%) was mostly GCB type. In parallel analysis, following numeralization of BCL2 DHL, BCL6 DHL and THL as 1, 2 and 3 , respectively, as well as 0 and 1 for non-GCB and GCB phenotypes, respectively, results of our paired $t$-test analysis showed a t-ratio of -6.53 and a correlation index of -1.70 , indicating a reversed effect directionality; thus by inference patients with BCL2 DHL were GCB type, while our BCL6 DHL and THL were mostly non-GCB type (Figure 2). 


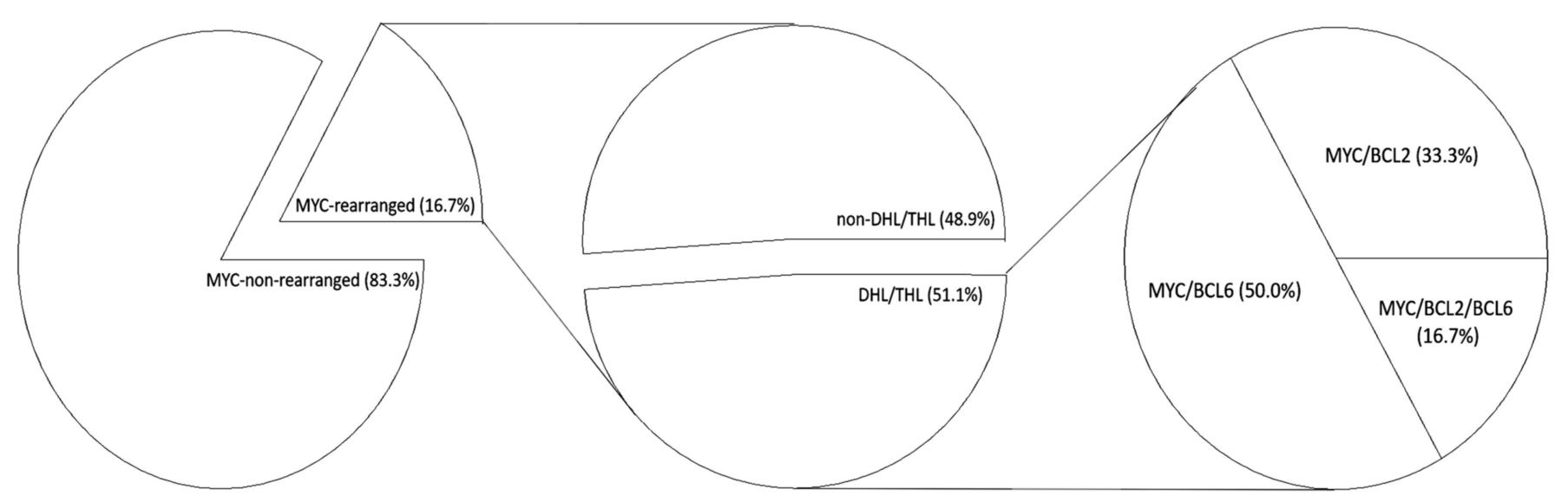

Figure 1. Pie-charts showing our DLBCL cohort $(n=282)$ distribution based on the presence or absence of MYCrearrangement (left), and multiple hit status (middle), as well as the stratification of the multiple hit group based on concurrent BCL2 and/or BCL6 rearrangements (right). The ratio of rearranged BCL2:BCL6 was 2:3. DHL, double hit lymphoma; THL, triple hit lymphoma.

A

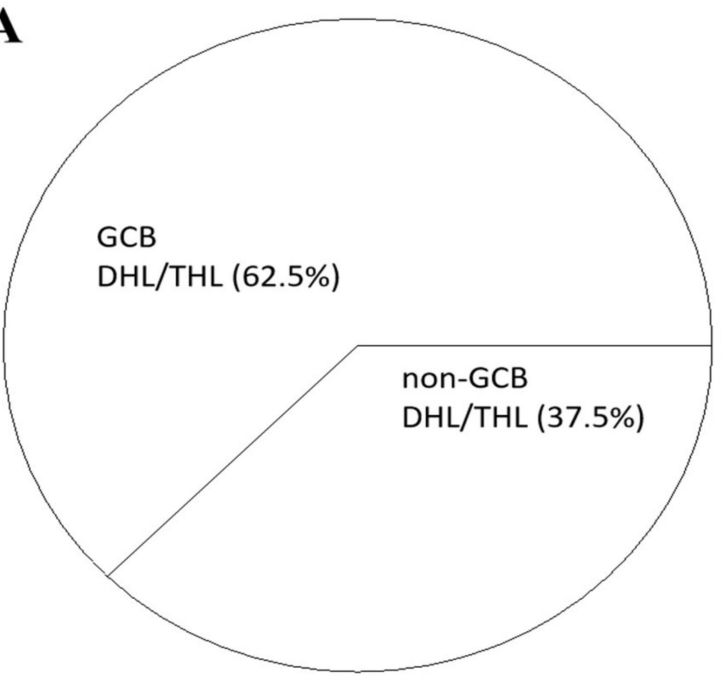

B

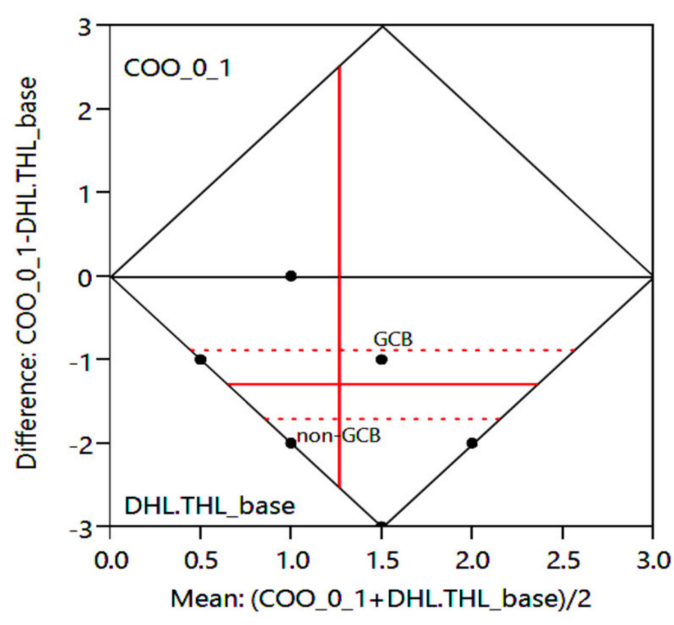

\begin{tabular}{llll}
\hline COO_0_1 & 0.61905 & t-Ratio & -6.52929 \\
DHL.THL_base & 1.90476 & Prob $>|\mathbf{t}|$ & $<.0001$ \\
Mean Difference & -1.2857 & Prob $>$ t & 1.0000 \\
Std Error & 0.19691 & Prob $<$ t & $<.0001$ \\
Upper 95\% & -0.875 & Correlation & -0.1093 \\
Lower 95\% & -1.6965 & & \\
\hline
\end{tabular}

Figure 2. (A) Pie-chart showing the COO-based constitution of our high-grade B-cell lymphoma (HGBL) with MYC and $B C L 2$ and/or BCL6 rearrangements $(n=24)$. (B) Chart showing the difference between the means of our high-grade B-cell lymphoma (HGBL) with MYC and BCL2 and/or BCL6 rearrangements (DHL/THL) and their COO. DHL, double hit lymphoma; THL, triple hit lymphoma; DEL, double expressor lymphoma; TEL, triple expressor lymphoma; N, sample size; $\mathrm{DF}$, degree of freedom; GCB, germinal center B-cell type; COO, cell-of-origin.

\subsubsection{MYC, BCL2, BCL6 Rearrangement and Expression}

Tables 1 and 2 summarize the relationships between MYC, BCL2 or BCL6 gene rearrangement and protein expression. Patients with $M Y C$ rearrangement alone were more likely to express MYC protein (73.9\%) than their counterparts with normal MYC gene (48.9\%); thus, compared with the patients with unaltered MYC gene, or those with concomitant MYC and BCL6 rearrangement (41.7\%), those harboring concurrent MYC and BCL2 rearrangements more frequently expressed MYC protein $(75.0 \%)$. More so, patients with rearranged BCL2 almost always expressed BCL2 protein $(87.5 \%)$ compared with those with 
MYC-only rearrangement (56.5\%) or rearranged MYC and BCL6 but normal BCL2 genes (58.3\%). Interestingly, we observed that all patients with THL, $75.0 \%$ of patients with BCL6 DHL, and $62.5 \%$ of $B C L 2$ DHL cases were BCL6 expressors. From comparative analysis using the paired $t$-test, we found the mean difference between patients with DHL/THL and DEL/TEL was $0.538 \pm 0.144$ (95\%CI: $0.225-0.852)$ with a t-ratio of 3.742 which is statistically significantly different from 0 at the $95 \% \mathrm{CI}$ (Prob $>t=0.003$; Prob $>t=0.001$; Prob $<t=0.999$ ), and correlation of 0 (Figure $3 \mathrm{~A}$ ), indicating that double or triple expressors (DEL/TEL) do not necessarily harbor double or triple hits (DHL/THL).

Table 1. Clinicopathological characteristics of our DLBCL cohort $(n=282)$ based on the presence or absence of gene rearrangement.

\begin{tabular}{|c|c|c|c|c|c|}
\hline & & \multicolumn{4}{|c|}{ Gene Rearrangement/Translocation } \\
\hline & & $\begin{array}{c}\text { Non-DHL/THL } \\
(\text { Non-MYC + (BCL2 } \pm \text { BCL6)) } \\
N=258(91.5 \%)\end{array}$ & $\begin{array}{c}\text { DHL/THL } \\
(\mathrm{MYC}+(\mathrm{BCL} 2 \pm \mathrm{BCL} 6)) \\
N=24(8.5 \%)\end{array}$ & $\begin{array}{c}\text { MYC Only } \\
N=23(8.2 \%)\end{array}$ & $p$-Value \\
\hline \multirow{2}{*}{\multicolumn{2}{|c|}{ Median Age \pm SD (years) }} & $66 \pm 15.42$ & $67.5 \pm 12.43$ & $64 \pm 16.93$ & \multirow{4}{*}{0.651} \\
\hline & & & & & \\
\hline & $<60$ & $83(32.2 \%)$ & $7(29.2 \%)$ & $10(43.5 \%)$ & \\
\hline & $\geq 60$ & $175(67.8 \%)$ & $17(70.8 \%)$ & $13(56.5 \%)$ & \\
\hline Ann-Arbor stage ( & & & & & 0.511 \\
\hline & Early (I/II) & $106(41.1 \%)$ & $9(37.5 \%)$ & $11(47.8 \%)$ & \\
\hline & Advanced (III/IV) & $152(58.9 \%)$ & $15(62.5 \%)$ & $12(52.2 \%)$ & \\
\hline $\operatorname{Sex}(\%)$ & & & & & 0.345 \\
\hline & Male & $146(56.6 \%)$ & $16(66.7 \%)$ & $14(60.9 \%)$ & \\
\hline & Female & $112(43.4 \%)$ & $8(33.3 \%)$ & $9(39.1 \%)$ & \\
\hline Ki67 expression & & & & & 0.789 \\
\hline & $<70 \%$ & $60(23.3 \%)$ & $6(25.0 \%)$ & $3(13.0 \%)$ & \\
\hline & $\geq 70 \%$ & $198(76.7 \%)$ & $18(75.0 \%)$ & $20(87.0 \%)$ & \\
\hline CD10 expression & & & & & 0.003 \\
\hline & Positive & $79(30.6 \%)$ & $15(62.5 \%)$ & $10(43.5 \%)$ & \\
\hline & Negative & $179(69.4 \%)$ & $9(37.5 \%)$ & $13(56.5 \%)$ & \\
\hline MUM1 expression & & & & & 0.003 \\
\hline & Positive & $202(78.3 \%)$ & $13(54.2 \%)$ & $14(60.9 \%)$ & \\
\hline & Negative & $56(21.7 \%)$ & $11(45.8 \%)$ & $9(39.1 \%)$ & \\
\hline MYC expression & & & & & 0.658 \\
\hline & Positive & $131(50.8 \%)$ & $14(58.3 \%)$ & $17(73.9 \%)$ & \\
\hline & Negative & $127(49.2 \%)$ & $10(41.7 \%)$ & $6(26.1 \%)$ & \\
\hline BCL2 expression & & & & & 0.026 \\
\hline & Positive & $215(83.3 \%)$ & $16(66.7 \%)$ & $13(56.5 \%)$ & \\
\hline & Negative & $43(16.6 \%)$ & $8(33.3 \%)$ & $10(43.5 \%)$ & \\
\hline BCL6 expression & & & & & 0.051 \\
\hline & Positive & $182(70.5 \%)$ & $18(75.0 \%)$ & $13(56.5 \%)$ & \\
\hline & Negative & $76(29.5 \%)$ & $6(25.0 \%)$ & $10(43.5 \%)$ & \\
\hline IPI score & & & & & 0.674 \\
\hline & $0-1$ & $81(31.4 \%)$ & $5(20.8 \%)$ & $10(43.5 \%)$ & \\
\hline & 2 & $57(22.1 \%)$ & $9(37.5 \%)$ & $3(13.0 \%)$ & \\
\hline & 3 & $48(18.6 \%)$ & $6(25.0 \%)$ & $6(26.1 \%)$ & \\
\hline & 4 & $43(16.7 \%)$ & $4(16.7 \%)$ & $1(4.3 \%)$ & \\
\hline & 5 & $29(11.2 \%)$ & $0(00.0 \%)$ & $3(13.0 \%)$ & \\
\hline Cell-of-Origin (CC & & & & & 0.007 \\
\hline & GCB & $101(39.1 \%)$ & $15(62.5 \%)$ & $12(52.2 \%)$ & \\
\hline & Non-GCB & $157(60.9 \%)$ & $9(37.5 \%)$ & $11(47.8 \%)$ & \\
\hline Follow-up time (d & $\mathrm{s}$, median $\pm \mathrm{SD}$ ) & $462.5 \pm 879.19$ & $484 \pm 753.31$ & $228 \pm 789.45$ & 0.883 \\
\hline
\end{tabular}


Table 2. MYC, BCL2 and BCL6 rearrangement-stratified clinicopathological characteristics of our DLBCL cohort $(n=282)$ based on the presence or absence of gene rearrangement.

MYC + (BCL2 \pm BCL6)

Gene Rearrangement/Translocation

\begin{tabular}{|c|c|c|c|c|c|}
\hline & & $\begin{array}{c}\text { MYC + BCL2 + BCL6 } \\
\quad N=4(16.7 \%)\end{array}$ & $\begin{array}{l}\text { MYC + BCL2 } \\
N=8(33.3 \%)\end{array}$ & $\begin{array}{c}\text { MYC + BCL6 } \\
N=12(50.0 \%)\end{array}$ & $p$-Value \\
\hline \multirow{2}{*}{\multicolumn{2}{|c|}{$\begin{array}{l}\text { Median Age } \pm \text { SD (Years) } \\
\text { Age }(\%)\end{array}$}} & $58 \pm 12.37$ & $77 \pm 10.77$ & $64.5 \pm 11.93$ & \\
\hline & & & 0.365 & & \\
\hline & $<60$ & $2(50.0 \%)$ & $1(12.5 \%)$ & $4(33.3 \%)$ & \\
\hline & $\geq 60$ & $2(50.0 \%)$ & $7(87.5 \%)$ & $8(66.7 \%)$ & \\
\hline \multirow[t]{3}{*}{ Ann-Arbor Stage (\% } & & & 0.856 & & \\
\hline & Early (I/II) & $2(50.0 \%)$ & $3(37.5 \%)$ & $6(50.0 \%)$ & \\
\hline & Advanced (III/IV) & $2(50.0 \%)$ & $5(62.5 \%)$ & $6(50.0 \%)$ & \\
\hline \multirow[t]{3}{*}{$\operatorname{Sex}(\%)$} & & & 0.296 & & \\
\hline & Male & $2(50.0 \%)$ & $7(87.5 \%)$ & $7(58.3 \%)$ & \\
\hline & Female & $2(50.0 \%)$ & $1(12.5 \%)$ & $5(41.7 \%)$ & \\
\hline \multirow[t]{3}{*}{ Ki67 Expression } & & & 0.069 & & \\
\hline & $<70 \%$ & $2(50.0 \%)$ & $4(50.0 \%)$ & 0 & \\
\hline & $\geq 70 \%$ & $2(50.0 \%)$ & $4(50.0 \%)$ & $12(100 \%)$ & \\
\hline \multirow[t]{3}{*}{ CD10 Expression } & & & 0.271 & & \\
\hline & Positive & $2(50.0 \%)$ & $7(87.5 \%)$ & $6(50.0 \%)$ & \\
\hline & Negative & $2(50.0 \%)$ & $1(12.5 \%)$ & $6(50.0 \%)$ & \\
\hline \multirow[t]{3}{*}{ MUM1 Expression } & & & 0.275 & & \\
\hline & Positive & $2(50.0 \%)$ & $2(25.0 \%)$ & $8(66.7 \%)$ & \\
\hline & Negative & $2(50.0 \%)$ & $6(75.0 \%)$ & $4(33.3 \%)$ & \\
\hline \multirow[t]{3}{*}{ MYC Expression } & & & 0.322 & & \\
\hline & Positive & $3(75.0 \%)$ & $6(75.0 \%)$ & $5(41.7 \%)$ & \\
\hline & Negative & $1(25.0 \%)$ & $2(25.0 \%)$ & $7(58.3 \%)$ & \\
\hline \multirow[t]{3}{*}{ BCL2 Expression } & & & 0.376 & & \\
\hline & Positive & $2(50.0 \%)$ & $7(87.5 \%)$ & $7(58.3 \%)$ & \\
\hline & Negative & $2(50.0 \%)$ & $1(12.5 \%)$ & $5(41.7 \%)$ & \\
\hline \multirow[t]{3}{*}{ BCL6 Expression } & & & 0.295 & & \\
\hline & Positive & $4(100.0 \%)$ & $5(62.5 \%)$ & $9(75.0 \%)$ & \\
\hline & Negative & 0 & $3(37.5 \%)$ & $3(25.0 \%)$ & \\
\hline \multirow[t]{6}{*}{ IPI Score } & & & 0.132 & & \\
\hline & $0-1$ & 0 & $2(25.0 \%)$ & $3(25.0 \%)$ & \\
\hline & 2 & $2(50.0 \%)$ & 0 & $6(50.0 \%)$ & \\
\hline & 3 & $2(50.0 \%)$ & $3(37.5 \%)$ & $1(8.3 \%)$ & \\
\hline & 4 & 0 & $3(37.5 \%)$ & $1(8.3 \%)$ & \\
\hline & 5 & 0 & 0 & $1(8.3 \%)$ & \\
\hline \multirow[t]{3}{*}{ Cell-of-Origin (COO } & & & 0.351 & & \\
\hline & GCB & $3(75.0 \%)$ & $7(87.5 \%)$ & $5(41.7 \%)$ & \\
\hline & Non-GCB & $1(25.0 \%)$ & $1(12.5 \%)$ & $7(58.3 \%)$ & \\
\hline \multicolumn{2}{|c|}{ Follow-up time (days, median $\pm \mathrm{SD}$ ) } & $1381 \pm 598.33$ & $177 \pm 122.18$ & $598.5 \pm 853.70$ & 0.088 \\
\hline
\end{tabular}

\subsubsection{MYC, BCL2, BCL6, Sex, Age, and Survival}

We also observed that the male gender was more predisposed to DHL (BCL2 DHL: $87.5 \%, B C L 6$ DHL: $58.3 \%$ ), gender association was equivocal for patients with THL (Male: $50 \%$ vs. Female: $50 \%$ ). Patients with $B C L 2$ DHL were found to be older (median age: $77 \pm 10.8$ years) than their BCL6 DHL (median age: $64.5 \pm 11.9$ years) or THL (median age: $58 \pm 12.4$ years) counterparts. Howbeit contextually confounding, we found that with a median survival of $177 \pm 122.2$ days, patients with $B C L 2$ DHL exhibited worse overall survival compared to the BCL6 DHL (598.5 \pm 853.7 days) or THL (1381 \pm 598.3 days) group. 
A

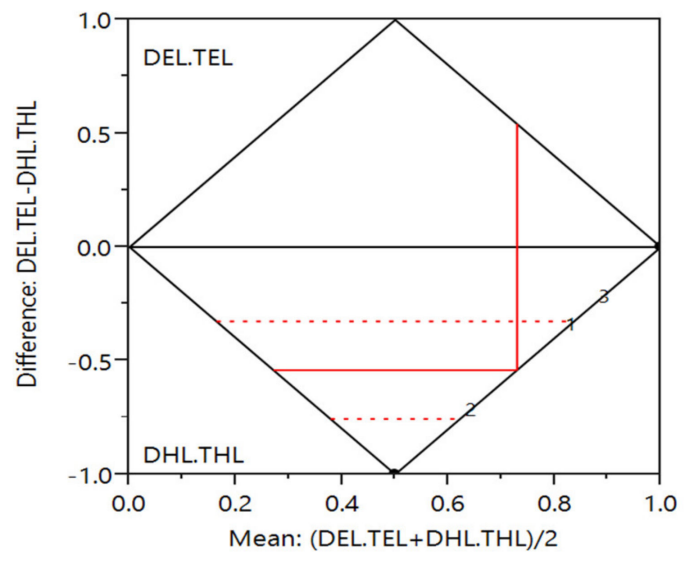

\begin{tabular}{llll}
\hline DEL.TEL & 0.45833 & t-Ratio & -5.21362 \\
DHL.THL & 1 & Prob $>|\mathbf{t}|$ & $<.0001$ \\
Mean Difference & -0.5417 & Prob $>$ t & 1.0000 \\
Std Error & 0.10389 & Prob $<$ t & $<.0001$ \\
Upper 95\% & -0.3267 & Correlation & 0 \\
Lower 95\% & -0.7566 & & \\
\hline
\end{tabular}

B

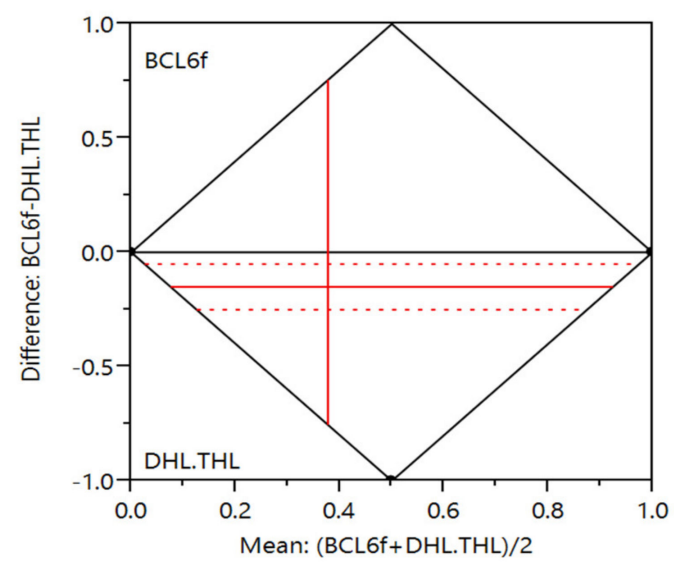

\begin{tabular}{lcll}
\hline BCL6f & 0.30189 & t-Ratio & -3.04047 \\
DHL.THL & 0.45283 & Prob > |t | & 0.0037 \\
Mean Difference & -0.1509 & Prob > t & 0.9982 \\
Std Error & 0.04964 & Prob < t & 0.0018 \\
Upper 95\% & -0.0513 & Correlation & 0.72286 \\
Lower 95\% & -0.2506 & & \\
\hline
\end{tabular}

Figure 3. Patients with DHL or THL mostly harbor BCL6 rearrangement, are mostly BCL6 expressors, but are not necessarily double or triple expressors. (A) Chart showing the difference between the means of our high-grade B-cell lymphoma (HGBL) with MYC and BCL2 and/or BCL6 rearrangements (DHL/THL) and those with MYC and BCL2 and/or BCL6 protein expression (DEL/TEL). (B) Chart showing the difference between the means of our high-grade B-cell lymphoma (HGBL) with MYC and BCL2 and/or BCL6 rearrangements (DHL/THL) and those with BCL6-rearrangement (BCL6f). DHL, double hit lymphoma; THL, triple hit lymphoma; DEL, double expressor lymphoma; TEL, triple expressor lymphoma; BCL6f, BCL6-rearranged.

\subsection{Gene Rearrangement, Protein Expression, and Proliferation Index}

Consistent with the suggestion that the median proliferation index of DHL approaches 90\% [11], as indicated in Tables 1 and 2, we found that added to a median Ki67 index of $90 \pm 6.41 \%$, All patients with BCL6 DHL had a Ki67-based proliferation index $\geq 70 \%$, compared with only $50.0 \%$ of the BCL2 DHL group, $87.0 \%$ from the MYC only-rearranged group or $76.7 \%$ of patients without multiple hits. Interestingly, results of our paired $t$-test showed a correlation of $72.3 \%$ between BCL6 rearrangement and DHL/THL status, with mean difference of $-0.15 \pm 0.05$ and $t$-ratio of -3.04 (Figure 3B). This data does suggest that while DLBCL cells are characteristically highly proliferative, a role for BCL6 as 'driver' or 'enhancer' of this highly proliferative phenotype especially in DHL cases cannot be disregarded. High proliferation index in the MYC only-rearranged and BCL6 DHL cases were mostly associated with low or equivocal CD10 expression and co-immunopositivity of MUM1, MYC, BCL2 and BCL6 protein expression, and these patients were more likely to be non-GCB type DLBCL.

\subsection{Prognostic Relevance of MYC, BCL2, or BCL6 Rearrangement and Expression}

The median follow-up duration for our whole cohort $(n=282)$ was 465 months, ranging from 0 to 3835 days, while it was $484 \pm 753.3$ days for patients with DHL/THL. Regardless of rearrangement status, there were $108(38.3 \%)$ recorded deaths; $22(7.8 \%)$ of these were secondary to non-DLBCL-related causes, 70 patients $(24.8 \%)$ were disease-specific mortality cases while the remaining 16 patients were without recorded cause of death. $8.5 \%$ $(24 / 282)$ of the whole DLBCL cohort were patients with documented recurrent disease. Survival analyses showed that patients with DHL/THL had shorter overall survival (OS) than their non-DHL/THL counterparts $\left(\chi^{2}=3.50\right.$, Prob $\left.>\chi^{2}=0.06\right)$ (Figure 4A). Upon stratification by hits, by the end of year 5, all patients with THL, BCL2 DHL and all but one BCL6 DHL cases had expired or were lost to follow-up $\left(\chi^{2}=8.56\right.$, Prob $\left.>\chi^{2}=0.01\right)$ 
(Figure 4B). When age-adjusted, for patients with DHL/THL, age $<60$ years $(n=90)$ conferred a survival advantage of 500 days ( 17 months) compared to those aged $\geq 60$ years $(n=192)$ (Figure 4C,D). Similarly, progression-free survival (PFS) was longer for the non-DHL/THL group compared with the DHL/THL group $\left(\chi^{2}=2.01\right.$, Prob $\left.>\chi^{2}=0.16\right)$ (Figure 4E,F); More so, while the six patients with BCL2 DHL were lost to follow-up by day 800, their remaining THL and BCL6 DHL peers exhibited very poor PFS, regardless of age strata (Figure 4F, Figure S2). Moreover, double and triple expressors (DEL/TEL) were less likely to be alive by day 2600 (86.7 month), compared with their non-DEL/TEL counterparts who enjoyed survival advantage of 900 days ( 30 months; $\chi^{2}=1.9$, Prob $\left.>\chi^{2}=0.16\right)$ (Figure S1A) and longer PFS $\left(\chi^{2}=1.15\right.$, Prob $\left.>\chi^{2}=0.28\right)$ (Figure S1B). We also observed 19 cases of extranodal involvement; $68.4 \%$ and $52.6 \%$ of which were BCL6-, and BCL2rearranged, respectively (Table S1). More than twice as much patients without BCL6 translocation $(n=6)$ harbored BCL6 rearrangement $(n=13)$ and exhibited extranodal involvement. Moreover, patients with BCL6 rearrangement were 5.5-fold more likely associated with extranodal involvement compared with their BCL2-rearranged peers ((BCL6: $\mathrm{HR}(95 \% \mathrm{CI})=3.8(0.64-22.2)$ vs. $B C L 2: \mathrm{HR}(95 \% \mathrm{CI})=0.6847(0.2265$ to 2.0701$))$, and our variable-outcome analysis indicate that while BCL2 rearrangement exhibited no statistically relevant relationship with extranodal involvement $\left(\chi^{2}=0.50, p=0.48\right), B C L 6$ hits showed a significantly strong relationship with extranodal involvement $\left(\chi^{2}=5.79, p=0.02\right)$ (Table S1).
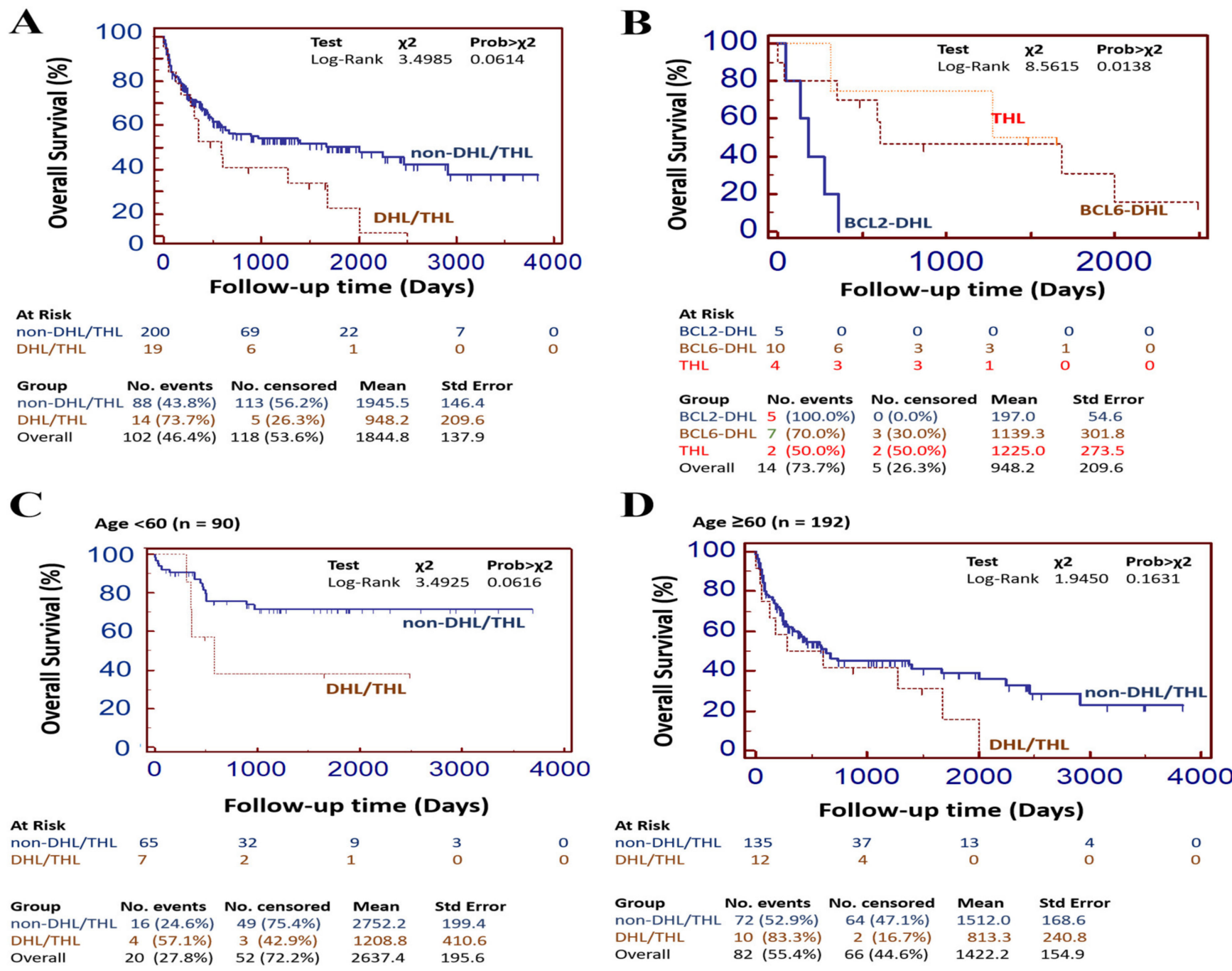

Figure 4. Cont. 

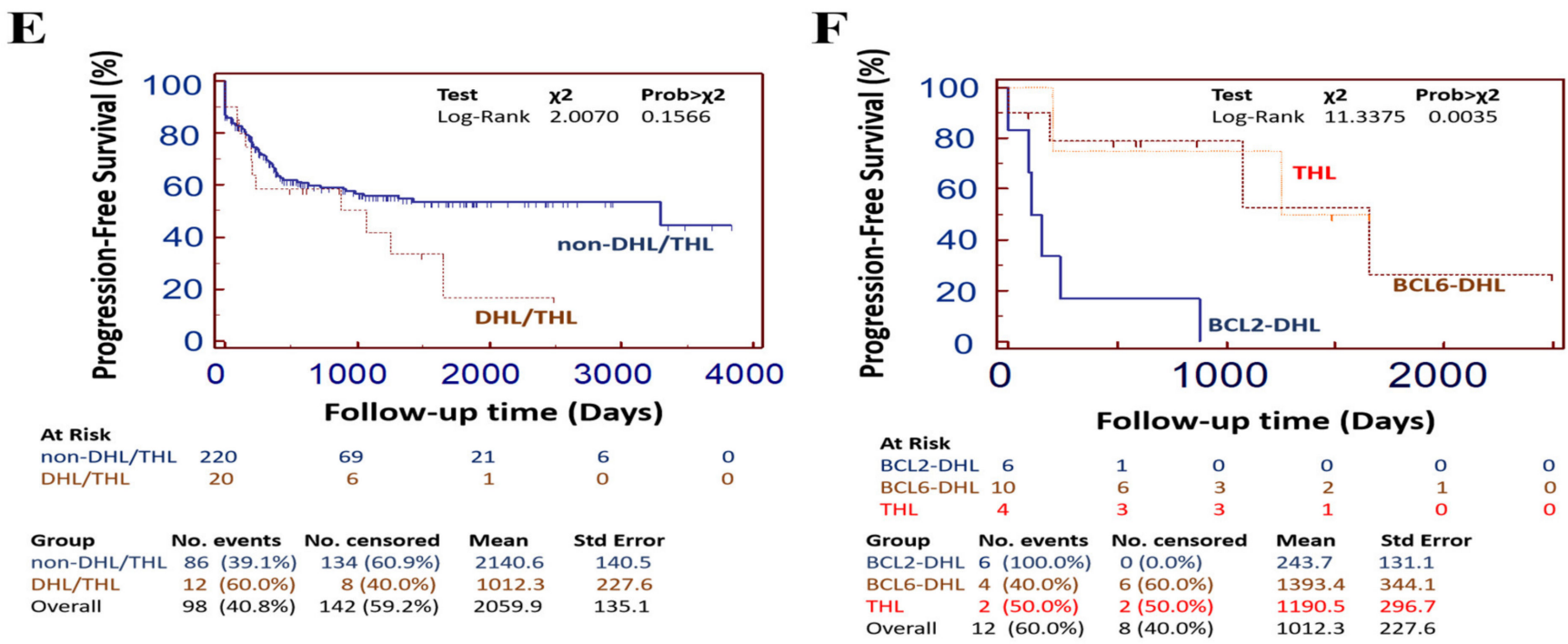

Figure 4. Multiple hits (DHL/THL) confer poor prognosis. Kaplan-Meier curves showing the differential effect of (A) DHL/THL or non-DHL/THL, and (B) BCL2-DHL, BCL6-DHL or THL on the overall survival of our DLBCL cohort ( $n=282)$. Kaplan-Meier curves showing the age-adjusted differential effect of DHL/THL or non-DHL/THL on the overall survival of our DLBCL cohort $(n=282)$ in patients $(C)$ younger than, or $(D)$ older than 60 years. Kaplan-Meier curves showing the differential effect of (E) DHL/THL or non-DHL/THL, and (F) BCL2-DHL, BCL6-DHL or THL on the Progression-free survival of our DLBCL cohort $(n=282)$. DHL, double hit lymphoma; THL, triple hit lymphoma; $X^{2}$, chi-square.

\section{Discussion}

DLBCL is a particularly aggressive disease entity which is broadly characterized by recurrent gene aberration, including the relatively infrequent presence of MYC (8q24), $B C L 2$ (18q21), and/or BCL6 (3q27) gene translocations/rearrangements [11-17]. Despite our increased understanding of DHL/THL biology and advances made in the therapeutic management of these therapy-evasive HGBL with MYC and BCL2 and/or BCL6 rearrangements, the nosological characterization of DHL/THL remains incomplete, necessitating transnational or multicenter large cohort studies to unravel relevant disease-specific genetic and clinicopathological features, as well as risk factors for appropriate characterization of risk-adapted therapeutic strategies [11-16,20]. More so, while acknowledging the poor prognostic peculiarity of DHL/THL and the benefits associated with inclusion of MYC, BCL2, and BCL6 gene translocation and protein expression in routine clinical work-up of patients with suspected DLBCL, little is known about the differential ethno-specific or geo-regional signature of DHL/THL. In fact, while the clinicopathological characteristics and probable therapeutic options of BCL2-rearranged DHL are well documented, only few studies have focused on BCL6-rearranged DHL and its clinical features.

Against the background of suggested geographic variation in the molecular pathogenesis of lymphoma [21], in the present study, exploring for probable Taiwan-specific clinicopathological and cytogenetic characteristics of DLBCL, we observed that $8.5 \%(24 / 282)$ of our DLBCL cohort were HGBL with re-arranged MYC and BCL2 and/or BCL6 genes, which is consistent with earlier FISH-based studies indicating that $7-10 \%$ patients with DLBCL harbor MYC, BLC2 and/or BCL6 rearrangement [11,13]; however compared with the estimated percentage in most published works $[11,13]$, we report a higher prevalence of MYC translocation prevalence in our cohort ( $10 \%$ vs. $16.5 \%)$.

Similar to the documented predominance of the GCB subtype in global DHL/THL cases (more specifically, from western countries) $[13,22,23]$, our Taiwanese DHL/THL cohort were predominantly GCB phenotype. Interestingly and of clinical relevance, the present study reports the predominance of BCL6-rearranged DHL and a 3-to-2 BCL6to- $B C L 2$-rearranged DHL ratio, in contrast to the broadly reported $B C L 2$ predominant DHL and the global $B C L 6$-to- $B C L 2$ ratio ranging from 1:1 to 1:8 [11]. We posit that this 
enhanced BCL6:BCL2 ratio and predominance of BCL6 rearrangement among patients with $\mathrm{DHL}$ is not only suggestive of a selective complementary role for MYC and BCL6 among Taiwanese patients, but may be associated with the poor therapy response and aggressive clinical course of our patients with DHL. This is even more so considering that these BCL6rearranged DHL were immunophenotypically unlike their BCL2 DHL counterpart, being principally non-GCB (immunoblastic, ABC type) in our cohort. This is consistent with reports indicating that patients with BCL6 DHL are less likely immunophenotypically GCB, clinically aggressive, characterized by poor OS, and that the "ABC DLBCL is associated with substantially worse outcomes when treated with standard chemoimmunotherapy", unlike their GCB peers with better therapeutic outcomes [14,24]. On the contrary, a number of studies (originating mostly from western countries) suggest that DLBCL "patients with either MYC/BCL6 rearrangements or MYC/BCL6 co-expression did not always have poorer prognosis", including report that BCL6-rearranged DHL cases are phenotypically GCB, and exhibit markedly better survival rates compared to their BCL2 DHL counterparts, who were found to be largely $\mathrm{ABC}$ in phenotype [25]. Interestingly, Li et al., using a similar Caucasian cohort as the study alluded above, found no apparent difference in the clinicopathological characteristics of patients with BCL6 DHL and those with BCL2 DHL, except that the BCL6 DHL group were less likely to be GCB in immunophenotype and had poor overall survival [14].

In addition, we observed that all our patients with BCL6 DHL had a Ki67 proliferation index $\geq 70$, compared with half of the BCL2 DHL group, suggesting a critical role for $B C L 6$ in the highly proliferative phenotype of our DHL cases. This finding is consistent with reports indicating that the constitutively pro-oncogenic BTB/POZ domain-containing BCL6, a transcriptional repressor of apoptosis, inflammation, and cell cycle control-effector genes [11], is required for B-cell proliferation, represses replication checkpoints, enhances tolerance to DNA damage [26,27]. Moreover, the high proliferation index of our BCL6 DHL cases is associated with low/equivocal CD10 expression and co-immunopositivity of MUM1, MYC, BCL2 and BCL6 protein expression, and these patients were more likely to be non-GCB type DLBCL. Concordant with accruing evidence, our finding highlights the essential role of BCL6 in the survival of DLBCL regardless of $C O O$, and perhaps explains why targeting BCL6 is equally efficacious for suppression of both the very aggressive non-GCB and relatively less aggressive GCB type DHL [28].

Our study also demonstrated that Taiwanese patients with BCL6 DHL are not necessarily MYC protein expressors, but are more often than not BCL6 expressors. Against the background that BCL2 and MYC are mostly co-expressed, our finding is congruent with Pillai RK et al.'s observation that unlike BCL2 DHL cases, BCL6 DHL are more likely to be CD10- but IRF4/MUM1+ and, more like Burkitt lymphoma, are cytogenetically less complex, and only infrequently express BCL2 [29], and by inference, less often express MYC. This may be associated with reported self-mitigating propensity of BCL6, a modulator of B cell receptor signals, to transcriptionally repress oncogenes such as MYC, BCL2, cyclin D1 (CCND1), and B lymphoma Mo-MLV Insertion Region 1 homolog (BMI1) [30,31]. It is thus evident and mechanistically relevant that BCL6 rearrangements are not associated with disruption of BCL6 coding sequence, but does replace the BCL6 promoter sites with other promoters; this allows BCL6 transformation-associated malignant transformation of B cells through dysregulated expression of normal BCL6 protein [30]. Concordant with the hypothesis of this present study, it is scientifically plausible that "because BCL6 represses beta-interferon gene positive-regulatory domain 1 binding factor (BLIMP1), which in turn represses $M Y C$, BCL6 translocations might be considered functionally equivalent to $M Y C$ translocation" [31]. Consistently, while our THL are equivocal for age or distribution, Ann-Arbor staging, Ki-67, CD10, MUM1, and BCL2 proteins expression, they are predominantly MYC expressors (75\%) and wholly BCL6 expressors (100\%). Thus, our study results portray BCL6 as a principal determinant of HGBL-DH/TH in Taiwan. The predominance of BCL6 rearrangement and associated aberrant expression of BCL6, BCL2, MUM1, and MYC proteins in Taiwanese patients with DHL in contrast to BCL6 DHL rarity in the West 
informs medical decision making, and does suggest that BCL6 is a promising therapeutic target $[32,33]$. There are reports demonstrating the druggability of BCL6 and the strong antiproliferative effect of its degradation [34], as well as evidence that the conditional deletion of BCL6 in DLBCL tumors in vivo induced significant inhibition of tumor growth with initial tumor stasis and subsequent attenuated tumor growth kinetics [35]. This is of contextual relevance and lends some credence to the findings of our present study which makes a case for the routine screening for BCL6 gene rearrangement by FISH and protein expression by IHC in all newly diagnosed DLBCL cases in Taiwan, and recommends the molecular or pharmacological targeting of BCL6 as an efficacious therapeutic strategy in managing patients with aggressive DHL regardless of COO in Taiwan.

It is worth mentioning that though initially confounding, the seemingly worse survival among BCL2 DHL in the present study is attributable to the larger number of their constituent patients in advanced stage with $62.5 \%$ stage III/IV and $37.5 \%$ stage I/II, compared with the BCL6 DHL group with equiproportional cases in early and late stages. More so, these patients with $B C L 2$ DHL were more advanced in age with median age of $77 \pm 10.8$ years. Aside these risk factor, consistent with the well documented role of BCL6 in the initiation, therapy evasion, and progression of hematological malignancies, including DLBCL [11,26-35], it is conceivable that this worse prognosis is associated with the high expression of BCL6 protein in most of these BCL2 DHL cases $(62.5 \%$ BCL6+ vs. $37.5 \%$ BCL6-). Thus, we posit that this observed poor prognosis may be attributable to aberrant BCL6 expression and not because of the BCL2 translocation per se, in the BCL2 DHL cases.

\section{Conclusions}

In conclusion, contrary to contemporary data from western countries, the majority of patients with multiple hits-DLBCL in Taiwan harbor BCL6 rearrangement. While IHC-based co-overexpression of MYC and BCL2 proteins is a good surrogate for BCL2rearranged DHL, same may not be said for BCL6-rearranged DH/TH-DLBCL in Taiwan. More than half of our BCL6-rearranged DH-DLBCL cases were non-GCB phenotype, indicating, at least in part, that the preferential screening for DH with BCL6 rearrangement may be a clinically-informative modality for patients with the non-GCB phenotype DLBCL in Taiwan. Consistent with present findings, we recommend mandatory screening for BCL6-rearranged DH DLBCL in Taiwan.

Supplementary Materials: The following are available online at https:/ / www.mdpi.com/article/ 10.3390/cancers13071620/s1, Table S1: Extranodal Involvement. Figure S1: Multi-gene expression (DEL/TEL) confer poor prognosis. Figure S2: Age-adjusted survival analysis.

Author Contributions: Study conception and design: C.-C.T., Y.-C.S., O.A.B., T.-Y.C. Collection and assembly of data: C.-C.T., Y.-C.S., Y.-Y.H., W.-H.C., C.-H.L., S.-M.H., M.-L.L., C.-L.F., C.-L.C. (Chi-Long Chen), C.-L.C. (Chia-Lun Chang), C.-T.Y., Y.-M.Z., H.-T.L., W.-H.L., B.-J.C., W.-C.T., H.-W.L., S.-M.H., C.-L.H., S.-W.L., H.-E.T., Y.Y., J.W.-P., T.-Y.C. Data analysis and interpretation, manuscript writing: C.-C.T., O.A.B., Y.-C.S., T.-Y.C. Provision of study resources and administrative oversight: O.A.B., Y.Y., J.W.-P., T.-Y.C. All authors have read and agreed to the published version of the manuscript.

Funding: This work was financially supported by the Higher Education Sprout Project grant of the Taiwan Ministry of Education (MOE) (DP2-108-21121-01-C-02-02) to Yun Yen, Huey-En Tzeng, and Tsu-Yi Chao.

Institutional Review Board Statement: This study was conducted according to the guidelines of the Declaration of Helsinki and approved by the Institutional Human Research Ethics Review Board (TMU-JIRB No. 201908048, Date of approval: 31.08.2019) of Taipei Medical University.

Informed Consent Statement: The need for consent to participate was waived because of the retrospective nature of the study. 
Data Availability Statement: The data presented in this study are available on request from the corresponding author. The data are not publicly available due to patient confidentiality and institutional data publication policy.

Acknowledgments: The authors would like to acknowledge the contribution of our late colleague Yung-Cheng Su who actually conceived and initiated this study before his demise last year 2019. We also thank Lin Yen-Kuang of the Research Center of Statistics, Taipei Medical University, for his valuable guidance on statistical methods. We would also like to appreciate the Academic editors, the reviewers, and the Cancers editorial team for their constructive critiques and suggestions, as well as their stellar professionalism from sublission to final acceptance and publication of this present work.

Conflicts of Interest: The authors declare that they have no potential conflicting interests.

\section{References}

1. Chuang, S.S.; Chen, S.W.; Chang, S.T.; Kuo, Y.T. Lymphoma in Taiwan: Review of 1347 neoplasms from a single institution according to the 2016 Revision of the World Health Organization Classification. J. Formos. Med. Assoc. 2017, 116, 620-625. [CrossRef]

2. Hans, C.P.; Weisenburger, D.D.; Greiner, T.C.; Gascoyne, R.D.; Delabie, J.; Ott, G.; Müller-Hermelink, H.K.; Campo, E.; Braziel, R.M.; Jaffe, E.S.; et al. Confirmation of the molecular classification of diffuse large B-cell lymphoma by immunohistochemistry using a tissue microarray. Blood 2004, 103, 275-282. [CrossRef]

3. Liu, Y.; Barta, S.K. Diffuse large B-cell lymphoma: 2019 update on diagnosis, risk stratification, and treatment. Am. J. Hematol. 2019, 94, 604-616. [CrossRef]

4. Crombie, J.L.; Armand, P. Diffuse Large B-Cell Lymphoma's New Genomics: The Bridge and the Chasm. J. Clin. Oncol. 2020, 38, 3565-3574. [CrossRef]

5. Scott, D.W.; Mottok, A.; Ennishi, D.; Wright, G.W.; Farinha, P.; Ben-Neriah, S.; Kridel, R.; Barry, G.S.; Hother, C.; Abrisqueta, P.; et al. Prognostic Significance of Diffuse Large B-Cell Lymphoma Cell of Origin Determined by Digital Gene Expression in Formalin-Fixed Paraffin-Embedded Tissue Biopsies. J. Clin. Oncol. 2015, 33, 2848-2856. [CrossRef]

6. Staiger, A.M.; Ziepert, M.; Horn, H.; Scott, D.W.; Barth, T.F.; Bernd, H.W.; Feller, A.C.; Klapper, W.; Szczepanowski, M.; Hummel, M.; et al. German High-Grade Lymphoma Study Group. Clinical Impact of the Cell-of-Origin Classification and the MYC/BCL2 Dual Expresser Status in Diffuse Large B-Cell Lymphoma Treated Within Prospective Clinical Trials of the German High-Grade Non-Hodgkin's Lymphoma Study Group. J. Clin. Oncol. 2017, 35, 2515-2526.

7. Monti, S.; Savage, K.J.; Kutok, J.L.; Feuerhake, F.; Kurtin, P.; Mihm, M.; Wu, B.; Pasqualucci, L.; Neuberg, D.; Aguiar, R.C.; et al. Molecular profiling of diffuse large B-cell lymphoma identifies robust subtypes including one characterized by host inflammatory response. Blood 2005, 105, 1851-1861. [CrossRef] [PubMed]

8. Schmitz, R.; Wright, G.W.; Huang, D.W.; Johnson, C.A.; Phelan, J.D.; Wang, J.Q.; Roulland, S.; Kasbekar, M.; Young, R.M.; Shaffer, A.L.; et al. Genetics and Pathogenesis of Diffuse Large B-Cell Lymphoma. N. Engl. J. Med. 2018, 378, $1396-1407$. [CrossRef] [PubMed]

9. Alizadeh, A.A.; Eisen, M.B.; Davis, R.E.; Ma, C.; Lossos, I.S.; Rosenwald, A.; Boldrick, J.C.; Sabet, H.; Tran, T.; Yu, X.; et al. Distinct types of diffuse large B-cell lymphoma identified by gene expression profiling. Nature 2000, 403, 503-511. [CrossRef] [PubMed]

10. Wiestner, A.; Staudt, L.M. Towards molecular diagnosis and targeted therapy of lymphoid malignancies. Semin. Hematol. 2003, 40, 296-307. [CrossRef]

11. Aukema, S.M.; Siebert, R.; Schuuring, E.; van Imhoff, G.W.; Kluin-Nelemans, H.C.; Boerma, E.J.; Kluin, P.M. Double-hit B-cell lymphomas. Blood 2011, 117, 2319-2331.

12. Green, T.M.; Young, K.H.; Visco, C.; Xu-Monette, Z.Y.; Orazi, A.; Go, R.S.; Nielsen, O.; Gadeberg, O.V.; Mourits-Andersen, T.; Frederiksen, M.; et al. Immunohistochemical double-hit score is a strong predictor of outcome in patients with diffuse large B-cell lymphoma treated with rituximab plus cyclophosphamide, doxorubicin, vincristine, and prednisone. J. Clin. Oncol. 2012, 30, 3460-3467.

13. Rosenthal, A.; Younes, A. High grade B-cell lymphoma with rearrangements of MYC and BCL2 and/or BCL6: Double hit and triple hit lymphomas and double expressing lymphoma. Blood Rev. 2017, 31, 37-42. [CrossRef] [PubMed]

14. Li, S.; Desai, P.; Lin, P.; Yin, C.C.; Tang, G.; Wang, X.J.; Konoplev, S.N.; Khoury, J.D.; Bueso-Ramos, C.E.; Medeiros, L.J. MYC/BCL6 double-hit lymphoma (DHL): A tumour associated with an aggressive clinical course and poor prognosis. Histopathology 2016, 68, 1090-1098. [CrossRef] [PubMed]

15. Li, S.; Lin, P.; Medeiros, L.J. Advances in pathological understanding of high-grade B cell lymphomas. Expert Rev. Hematol. 2018, 11, 637-648.

16. Sabattini, E.; Bacci, F.; Sagramoso, C.; Pileri, S.A. WHO classification of tumours of haematopoietic and lymphoid tissues in 2008: An overview. Pathologica 2010, 102, 83-87.

17. Choi, S.M.; O'Malley, D.P. Diagnostically relevant updates to the 2017 WHO classification of lymphoid neoplasms. Ann. Diagn. Pathol. 2018, 37, 67-74. [CrossRef]

18. Quesada, A.E.; Medeiros, L.J.; Desai, P.A.; Lin, P.; Westin, J.R.; Hawsawi, H.M.; Wei, P.; Tang, G.; Seegmiller, A.C.; Reddy, N.M.; et al. Increased MYC copy number is an independent prognostic factor in patients with diffuse large B-cell lymphoma. Mod. Pathol. 2017, 30, 1688-1697. [CrossRef] [PubMed] 
19. Huang, S.; Nong, L.; Wang, W.; Liang, L.; Zheng, Y.; Liu, J.; Li, D.; Li, X.; Zhang, b.; Li, T. Prognostic impact of diffuse large B-cell lymphoma with extra copies of MYC, BCL2 and/or BCL6: Comparison with double/triple hit lymphoma and double expressor lymphoma. Diagn. Pathol. 2019, 14, 81. [CrossRef] [PubMed]

20. Lindsley, R.C.; LaCasce, A.S. Biology of double-hit B-cell lymphomas. Curr. Opin. Hematol. 2012, 19, 299-304. [CrossRef] [PubMed]

21. Biagi, J.J.; Seymour, J.F. Insights into the molecular pathogenesis of follicular lymphoma arising from the analysis of geographic variation. Blood 2002, 99, 4265-4275. [CrossRef] [PubMed]

22. Visco, C.; Tzankov, A.; Xu-Monette, Z.Y.; Miranda, R.N.; Tai, Y.C.; Li, Y.; Liu, W.M.; d'Amore, E.S.; Li, Y.; Montes-Moreno, S.; et al. Patients with diffuse large B-cell lymphoma of germinal center origin with BCL2 translocations have poor outcome, irrespective of MYC status: A report from an International DLBCL rituximab-CHOP Consortium Program Study. Haematologica 2013, 98, 255-263. [CrossRef] [PubMed]

23. Hu, S.; Xu-Monette, Z.Y.; Tzankov, A.; Green, T.; Wu, L.; Balasubramanyam, A.; Liu, W.M.; Visco, C.; Li, Y.; Miranda, R.N.; et al. MYC/BCL2 protein coexpression contributes to the inferior survival of activated B-cell subtype of diffuse large B-cell lymphoma and demonstrates high-risk gene expression signatures: A report from The International DLBCL Rituximab-CHOP Consortium Program. Blood 2013, 121, 4021-4031. [CrossRef] [PubMed]

24. Nowakowski, G.S.; Czuczman, M.S. ABC, GCB, and Double-Hit Diffuse Large B-Cell Lymphoma: Does Subtype Make a Difference in Therapy Selection? Am. Soc. Clin. Oncol. Educ. Book 2015, 35, e449-e457. [CrossRef] [PubMed]

25. Ye, Q.; Xu-Monette, Z.Y.; Tzankov, A.; Deng, L.; Wang, X.; Manyam, G.C.; Visco, C.; Montes-Moreno, S.; Zhang, L.; Dybkær, K.; et al. Prognostic impact of concurrent MYC and BCL6 rearrangements and expression in de novo diffuse large B-cell lymphoma. Oncotarget 2016, 7, 2401-2416. [CrossRef]

26. Basso, K.; Dalla-Favera, R. Roles of BCL6 in normal and transformed germinal center B cells. Immunol. Rev. 2012, $247,172-183$. [CrossRef] [PubMed]

27. Cerchietti, L.; Melnick, A. Targeting BCL6 in diffuse large B-cell lymphoma: What does this mean for the future treatment? Expert Rev. Hematol. 2013, 6, 343-345. [CrossRef]

28. McCoull, W.; Cheung, T.; Anderson, E.; Barton, P.; Burgess, J.; Byth, K.; Cao, Q.; Castaldi, M.P.; Chen, H.; Chiarparin, E.; et al. Development of a Novel B-Cell Lymphoma 6 (BCL6) PROTAC To Provide Insight into Small Molecule Targeting of BCL6. ACS Chem. Biol. 2018, 13, 3131-3141. [CrossRef]

29. Pillai, R.K.; Sathanoori, M.; Van Oss, S.B.; Swerdlow, S.H. Double-hit B-cell lymphomas with BCL6 and MYC translocations are aggressive, frequently extranodal lymphomas distinct from BCL2 double-hit B-cell lymphomas. Am. J. Surg. Pathol. 2013, 37, 323-332. [CrossRef]

30. Cardenas, M.G.; Oswald, E.; Yu, W.; Xue, F.; MacKerell, A.D., Jr.; Melnick, A.M. The Expanding Role of the BCL6 Oncoprotein as a Cancer Therapeutic Target. Clin. Cancer Res. 2017, 23, 885-893. [CrossRef]

31. Shaffer, A.L.; Yu, X.; He, Y.; Boldrick, J.; Chan, E.P.; Staudt, L.M. BCL-6 represses genes that function in lymphocyte differentiation, inflammation, and cell cycle control. Immunity 2000, 13, 199-212. [CrossRef]

32. Leeman-Neill, R.J.; Bhagat, G. BCL6 as a therapeutic target for lymphoma. Expert Opin. Ther. Targets 2018, 22, 143-152. [CrossRef]

33. Cortiguera, M.G.; García-Gaipo, L.; Wagner, S.D.; Leon, J.; Batlle-Lopez, A.; Delgado, M.D. Suppression of BCL6 function by HDAC inhibitor mediated acetylation and chromatin modification enhances BET inhibitor effects in B-cell lymphoma cells. Sci. Rep. 2019, 9, 16495. [CrossRef] [PubMed]

34. Kerres, N.; Steurer, S.; Schlager, S.; Bader, G.; Berger, H.; Caligiuri, M.; Dank, C.; Engen, J.R.; Ettmayer, P.; Fischerauer, B.; et al. Chemically induced degradation of the oncogenic transcription factor BCL6. Cell Rep. 2017, 20, 2860-2875. [CrossRef]

35. Schlager, S.; Salomon, C.; Olt, S.; Albrecht, C.; Ebert, A.; Bergner, O.; Wachter, J.; Trapani, F.; Gerlach, D.; Voss, T.; et al. Inducible knock-out of BCL6 in lymphoma cells results in tumor stasis. Oncotarget 2020, 11, 875-890. [CrossRef] [PubMed] 